\title{
openheart Associations between left bundle branch block with different PR intervals, QRS durations, heart rates and the risk of heart failure: a register- based cohort study using ECG data from the primary care setting
}

Marc Meller Søndergaard (D) , ${ }^{1}$ Johannes Riis, ${ }^{1}{ }^{\text {Karoline Willum Bodker, }}{ }^{1}$
Steen Møller Hansen, ${ }^{1}$ Jesper Nielsen, ${ }^{1}$ Claus Graff, ${ }^{2}$ Adrian Holger Pietersen, ${ }^{3}$
Jonas Bille Nielsen, ${ }^{4,5}$ Bhupendar Tayal ${ }^{6}{ }^{6}$ Christoffer Polcwiartek, ${ }^{7}$
Christian Torp-Pedersen, ${ }^{8}$ Peter Soegaard, ${ }^{9}$ Kristian Hay Kragholm ${ }^{1,10}$

- Additional material is published online only. To view, please visit the journal online (http://dx.doi.org/10.1136/ openhrt-2020-001425).

To cite: Søndergaard MM, Riis J, Bodker KW, et al. Associations between left bundle branch block with different PR intervals, QRS durations, heart rates and the risk of heart failure: a registerbased cohort study using ECG data from the primary care setting. Open Heart 2021;8:e001425. doi:10.1136/ openhrt-2020-001425

Received 20 August 2020 Revised 26 November 2020 Accepted 1 December 2020

Check for updates

(c) Author(s) (or their employer(s)) 2021. Re-use permitted under CC BY-NC. No commercial re-use. See rights and permissions. Published by BMJ.

For numbered affiliations see end of article.

Correspondence to Marc Meller Søndergaard; marcm-s12@hotmail.com

\section{ABSTRACT}

Aim Left bundle branch block (LBBB) is associated with an increased risk of heart failure (HF). We assessed the impact of common ECG parameters on this association using large-scale data.

Methods and results Using ECGs recorded in a large primary care population from 2001 to 2011 , we identified HF-naive patients with a first-time LBBB ECG. We obtained information on sex, age, emigration, medication, diseases and death from Danish registries. We investigated the association between the PR interval, QRS duration, and heart rate and the risk of HF over a 2-year follow-up period using Cox regression analysis.

Of 2471 included patients with LBBB, 464 (18.8\%) was found between $Q R S$ duration and heart rate $(p<0.01)$, and the analyses were stratified on these parameters. Using a QRS duration $<150$ ms and a heart rate $<70$ beats per minute (bpm) as the reference, all groups were statistically significantly associated with the development of HF. Patients with a QRS duration $\geq 150$ ms and heart rate $\geq 70 \mathrm{bpm}$ had the highest risk of developing HF (HR 3.17 (95\% $\mathrm{Cl} 2.41$ to $4.18, \mathrm{p}<0.001)$. There was no association between the PR interval and HF after adjustment.

Conclusion Prolonged QRS duration and higher heart rate were associated with increased risk of HF among primary care patients with LBBB, while no association was observed with PR interval. Patients with LBBB with both a prolonged QRS duration ( $\geq 150 \mathrm{~ms}$ ) and higher heart rate $(\geq 70 \mathrm{bpm})$ have the highest risk of developing HF.

\section{INTRODUCTION}

Left bundle branch block (LBBB) has an estimated prevalence of $0.19 \%-0.82 \%$ in the general population, varying between studies and increasing with age. ${ }^{12}$ LBBB is known to be associated with an increased risk of developed HF during follow-up. A significant interaction

\section{Key questions}

What is already known about this subject?

- It has been established that left bundle branch block is associated with an increased risk of heart failure. However, the underlying mechanisms are not fully understood.

What does this study add?

- We showed that patients with a longer QRS duration and higher heart rate have a higher risk of heart failure. We also showed that the PR interval was unrelated to heart failure.

How might this impact on clinical practice?

- Special attention should be paid to patients presenting with a longer QRS duration and higher heart rate in the primary care setting.

cardiovascular disease, including heart failure (HF) and long-term mortality. ${ }^{3-9}$ Patients with LBBB have an abnormal electrical activation due to conduction block resulting into delayed activation of the left ventricular (LV) free wall. ${ }^{10}$ This abnormal activation gradually leads to an inefficient contraction pattern with subsequent development of HF. ${ }^{10}$ However, since not all patients with LBBB develop HF, there must be other underlying associated pathophysiological and electrical factors which are playing a critical role in the eventual development of HF.

LBBB is a diagnosis which can be made with routine ECG recordings, thus identification of other common ECG parameters which can help in further risk stratification of these patients is important. 
Several studies of prognostic ECG markers in LBBB indicate that the duration of the QRS complex in patients with LBBB increases risk of developing $\mathrm{HF}^{6811}$ as it results in abnormal filling time of the ventricle. ${ }^{12}$ But the width of the QRS complex, in combination with other ECG parameters, has not been thoroughly examined. Two interesting parameters in this context are the PR interval and heart rate. Theoretically, a longer PR interval results in atrioventricular asynchrony impacting the ventricle filling which may result in HF, but it is not known if the PR interval affects the outcome in patients with LBBB. ${ }^{13}$ Similarly, an increase in heart rate decreases the ventricular filling time. Moreover, reflex tachycardia is observed in patients with deteriorating left ventricle function and HF, and could therefore be a marker of the beginning of $\mathrm{HF}^{14}$

We hypothesised that heart rate and QRS duration, as well as the PR interval in patients with LBBB, may be important risk markers that can optimise risk stratification and facilitate appropriate referral to secondary specialised cardiology evaluation and treatment. To investigate this, we identified primary care ECGs with LBBB and assessed the association between the heart rate and QRS duration as well as PR interval and the development of HF.

\section{METHODS}

\section{Study setting and population}

We studied 12-lead ECGs recorded between January 2001 and September 2011 at the Copenhagen General Practitioners Laboratory, a centralised core facility offering paraclinical examinations including high-quality and standardised digital ECG procedures in patients referred from general practitioners in the capital region of Copenhagen, Denmark, which represents around 30\% of the Danish population.

First, we identified all patients with LBBB using the validated Marquette 12SL algorithm, V.21 (online supplemental material 1). ${ }^{15} 16$ We included patients at the time of their first ECG recording showing LBBB, as done previously. $^{17}$

From the included patients with LBBB, we excluded those with missing date of birth, ECGs of poor quality, implanted cardiac devices, certain arrhythmias and known HF (online supplemental table 2). Patients with the following arrhythmias were excluded: pace rhythm, atrial fibrillation, atrial flutter, Wolf-Parkinson-White, or second or third degree atrioventricular block. Patients with a PR interval $<120 \mathrm{~ms}$ or $\geq 400 \mathrm{~ms}$ were excluded to ensure that patients did not have undetected arrhythmias. Patients with a heart rate $\geq 100$ beats per minute (bpm) were excluded as we suspected that they might have undiagnosed atrial fibrillation.

Lastly, we excluded patients with prior HF diagnosis and patients treated with loop diuretics in two consecutive calendar quarters prior to LBBB diagnosis (online supplemental table 2).

\section{Data sources, study variables and definitions}

From the ECG recordings, we retrieved QRS duration, heart rate and PR interval. Participants were divided into groups based on clinical cut-offs described in the literature; that is, $150 \mathrm{~ms}$ for QRS duration and $200 \mathrm{~ms}$ for PR interval as the definition of first degree heart block. ${ }^{18}$ For heart rate within the normal reference range, participants were divided by the median rounded to nearest decile.

In addition to ECGs, we included patient demographics and clinical characteristics by linkage to a number of nationwide registries. All individuals with permanent residence in Denmark are given a unique Central Personal Registration number, which can be used to match data across registries. For a detailed description of registries used, please refer to the online supplemental material 1.

\section{Outcome measures}

Patients were followed for 2 years or until first HF event including fatal HF, death from other cause, emigration or end of study period on the 31st of December 2012, whichever came first. The primary study endpoint was the development of HF.

HF was defined as either a hospital admission, outpatient contact, an emergency room contact with an HF diagnosis or death from HF as done previously (online supplemental table 2). The HF diagnosis has previously been shown to have a sensitivity of $29 \%$ and specificity of $99 \% .{ }^{19}$ To increase sensitivity, the use of loop diuretics was added, as done previously (online supplemental table 2) ${ }^{19-21}$ The 10th revision of the International Statistical Classification of Diseases and Related Health Problems (ICD-10) codes for identifying $\mathrm{HF}$ are summarised by Schmidt $e t a l^{22}$

\section{Covariates}

The following comorbidities within 5 years prior to the first ECG showing LBBB were included: previous acute myocardial infarction, chronic ischaemic heart disease, valvular heart disease, pericardial disease, endocarditis, myocarditis, peripheral vascular disease, cerebrovascular disease, chronic lung disease, renal disease, chronic liver disease, malignant neoplasia, hypertension and diabetes (see online supplemental table 3 for ICD-10 codes).

Diabetes was identified by ICD-10 codes or treatment with glucose lowering drugs for diabetes or treatment with antihypertensive drugs for hypertension, as done previously. $^{22} 23$

Baseline medication was defined as dispensed prescriptions within 180 days prior to inclusion and consisted of cardiac glycosides, beta-blockers, ACE inhibitors and aldosterone antagonists (online supplemental table 3). ${ }^{24}$

\section{Statistical analysis}

Continuous variables were described using means and SDs when normally distributed, and median with 1 st and 3rd quartiles (Q1-Q3) when not. Normality of continuous variables was checked by visual inspection of histograms. Categorical variables were described using counts and percentages. Accordingly, Wilcoxon Mann-Whitney 


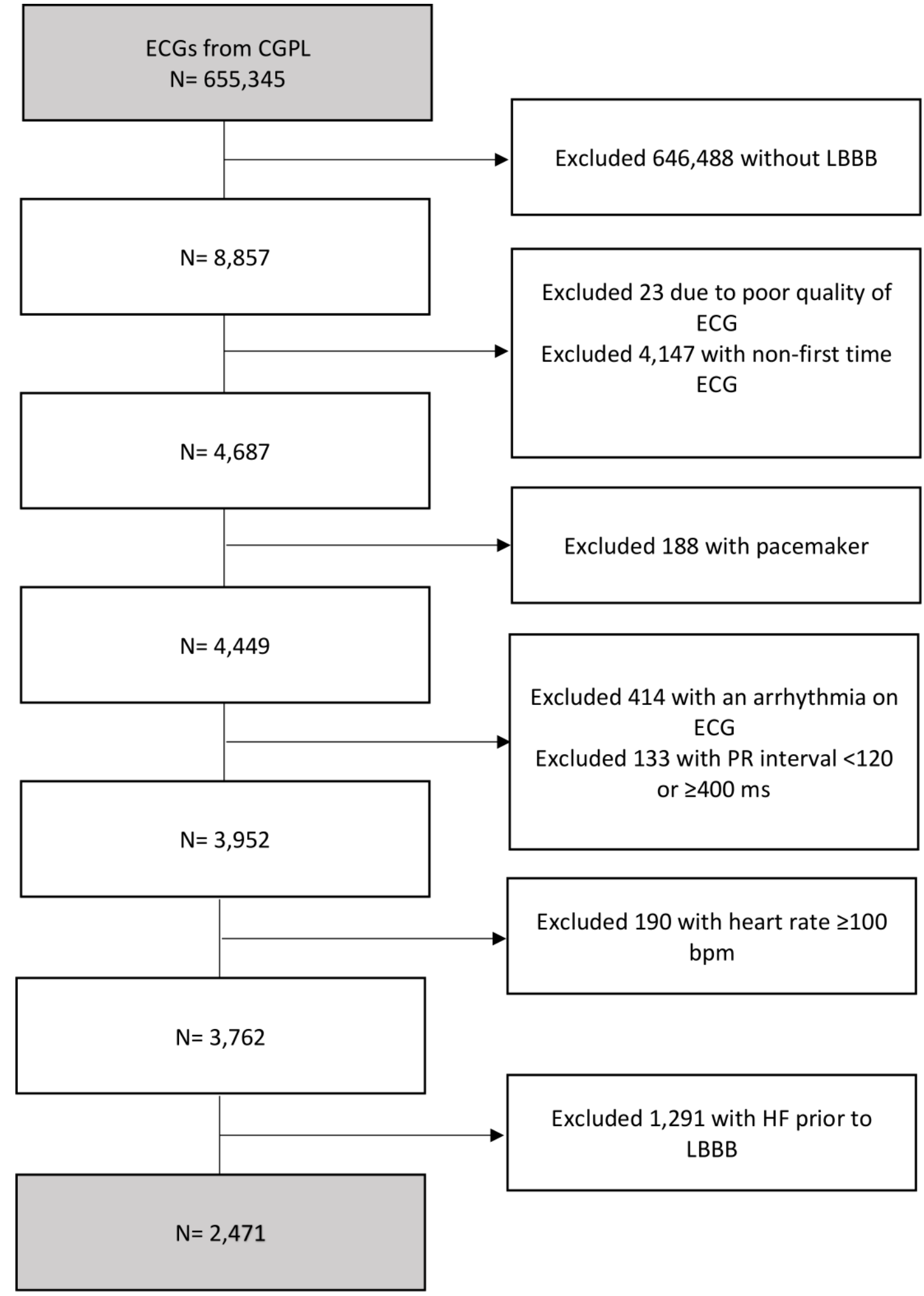

Figure 1 Patient selection flow chart. Selection of the study population. bpm, beats per minute; CGPL, Copenhagen General Practitioners Laboratory; HF, heart failure; LBBB, left bundle branch block.

(two groups) or Kruskal-Wallis (more than two groups) and Pearson's $\mathrm{X}^{2}$ tests were used to test for differences across exposure groups.

Aalen-Johansson cumulative incidence plots were made to assess the development of $\mathrm{HF}$ according to the different risk groups with death as a competing risk.
Univariate and multivariable Cox proportional hazards regressions were used to assess associations between QRS, PR and heart rate groups and the risk of developing HF.

Interaction analysis between exposures and all covariates were performed considering a $p$ value of $<0.01$ in a likelihood ratio test as statistically significant. In the case 


\begin{tabular}{|c|c|c|c|c|c|}
\hline Variable & $\begin{array}{l}\text { QRS }<150 \mathrm{~ms}+ \\
\text { heart rate }<70 \mathrm{bpm} \\
(\mathrm{n}=686)\end{array}$ & $\begin{array}{l}\text { QRS }<150 \mathrm{~ms}+ \\
\text { heart rate } \geq 70 \mathrm{bpm} \\
(\mathrm{n}=900)\end{array}$ & $\begin{array}{l}\text { QRS } \geq 150 \mathrm{~ms}+ \\
\text { heart rate }<70 \mathrm{bpm} \\
(\mathrm{n}=465)\end{array}$ & $\begin{array}{l}\text { QRS } \geq 150 \mathrm{~ms}+ \\
\text { heart rate } \geq 70 \mathrm{bpm} \\
(\mathrm{n}=420)\end{array}$ & $P$ value \\
\hline Age, median, Q1-Q3 & $72.2(62.8-80.8)$ & $74.4(64.7-82.1)$ & $70.3(60.4-78.5)$ & $70(61.1-78.6)$ & $<0.0001$ \\
\hline Male sex, no (\%) & $214(31.2)$ & $203(22.6)$ & $309(66.5)$ & $235(56.0)$ & $<0.001$ \\
\hline Hypertension, no (\%) & 269 (39.2) & $282(31.3)$ & $190(40.9)$ & $157(37.4)$ & $<0.001$ \\
\hline Diabetes, no (\%) & $36(5.2)$ & $66(7.3)$ & $39(8.4)$ & $44(10.5)$ & 0.01 \\
\hline MI, no (\%) & $11(1.6)$ & $8(0.9)$ & $8(1.7)$ & $<4$ & $(0.31)$ \\
\hline IHD w/o MI, no (\%) & $45(6.6)$ & $43(4.8)$ & $38(8.2)$ & $29(6.9)$ & 0.08 \\
\hline Malignancy, no (\%) & $42(6.1)$ & $58(6.4)$ & $23(4.9)$ & $12(2.9)$ & 0.05 \\
\hline PVD, no (\%) & $10(1.5)$ & $16(1.8)$ & $10(2.2)$ & $11(2.6)$ & 0.55 \\
\hline CVD, no (\%) & $29(4.2)$ & $34(3.8)$ & $23(4.9)$ & $18(4.3)$ & 0.79 \\
\hline CLD, no (\%) & $19(2.8)$ & $23(2.6)$ & $4(0.9)$ & $11(2.6)$ & 0.14 \\
\hline Liver disease, no (\%) & $<4$ & $<4$ & $<4$ & $<4$ & 0.54 \\
\hline Renal disease, no (\%) & $<4$ & $<4$ & $<4$ & $<4$ & 0.54 \\
\hline Cardiac glycosides, no (\%) & $8(1.2)$ & $10(1.1)$ & $4(0.9)$ & $4(1.0)$ & 0.96 \\
\hline ACE inhibitors, no (\%) & $137(20.0)$ & $83(9.2)$ & $101(21.7)$ & 48 (11.4) & 0.29 \\
\hline Class II anti-arrythmic & $8(1.2)$ & $5(0.6)$ & $3(0.6)$ & $6(1.4)$ & $<0.00$ \\
\hline AA, no (\%) & $8(1.2)$ & $5(0.6)$ & $<4$ & $6(1.4)$ & 0.33 \\
\hline Outcome (heart failure), no (\%) & $36(5.2)$ & $52(5.8)$ & $30(6.5)$ & $24(5.7)$ & \\
\hline Outcome (death), no (\%) & $72.2(62.8-80.8)$ & $74.4(64.7-82.1)$ & $70.3(60.4-78.5)$ & $70(61.1-78.6)$ & $<0.001$ \\
\hline
\end{tabular}

Data show characteristics according to QRS duration and heart rate. Subgroup 1: QRS duration $<150$ ms and heart rate $<70$ bpm. Subgroup 2: QRS duration $<150 \mathrm{~ms}$ and heart rate $\geq 70 \mathrm{bpm}$. Subgroup 3: QRS duration $\geq 150 \mathrm{~ms}$ and heart rate $<70 \mathrm{bpm}$. Subgroup 4: QRS duration $\geq 150 \mathrm{~ms}$ and heart rate $\geq 70 \mathrm{bpm}$. Categorical variables are presented with numbers and percentages and continuous variables with medians and $25 \%-75 \%$ percentiles (1st-3rd quartiles, Q1-Q3).

$\mathrm{AA}$, aldosterone antagonist; bpm, beats per minute; CLD, chronic lung disease; CVD, cerebrovascular disease; MI, myocardial infarction; IHD w/o MI, ischaemic heart disease without myocardial infarction; PVD, peripheral vascular disease.

of an interaction, stratification was applied using clinically relevant cut-offs.

The analyses for QRS groups were adjusted for age (quartiles), sex, heart rate $(<70 \mathrm{bpm}$ and $\geq 70 \mathrm{bpm})$, PR interval ( $<150 \mathrm{bpm}$ and $\geq 150 \mathrm{bpm})$, beta-blocker, hypertension, diabetes, previous acute myocardial infarction, chronic ischaemic heart disease and renal disease.

The analyses for the PR groups were adjusted for age (quartiles), sex, QRS duration, heart rate, beta-blocker, cardiac glycosides and calcium channel blockers.

In sensitivity analyses, we performed the same analyses with a combined endpoint of HF and death from other causes.

The proportional hazard assumptions were tested graphically using Schoenfeld residuals. Continuous relationships between the PR interval, QRS duration and HF were visualised using restricted cubic with three knots located at the 10th, 50th and 90th percentiles. These analyses were adjusted for the same covariates as the Cox regression analyses.

In all analyses, a p value of $<0.05$ was considered statistically significant.

Data management was performed using SAS, V.9.4 (SAS Institute), and statistical analysis was performed using RStudio, package 3.5.3, V.1.0.143 (RStudio Boston, Massachusetts, USA).

\section{RESULTS}

\section{Study population}

A total of 2471 patients were included in the study (figure 1), of whom 464 (18.8\%) developed HF while 142 $(5.7 \%)$ died during follow-up (table 1 ). The population was predominantly women $(61.1 \%)$ and the median age was $72.2,36.3 \%$ had hypertension and $7.5 \%$ had diabetes at baseline (table 1).

\section{QRS duration and heart rate groups}

Analyses revealed a significant interaction between the QRS groups and heart rate $(\mathrm{p}<0.01)$. Therefore, results for QRS duration and heart rate are only reported for stratified groups. Four subgroups were defined: (1) low heart rate and narrow QRS complex $(<70 \mathrm{bpm},<150 \mathrm{~ms}$, $\mathrm{n}=686$ ), (2) high heart rate and narrow QRS complex ( $\geq 70 \mathrm{bpm},<150 \mathrm{~ms}, \mathrm{n}=900$ ), (3) low heart rate and wide QRS complex $(<70 \mathrm{bpm}, \geq 150 \mathrm{~ms}, \mathrm{n}=465)$ and (4) high heart rate and wide QRS complex $(\geq 70 \mathrm{bpm}, \geq 150 \mathrm{~ms}$, $\mathrm{n}=420)$. 


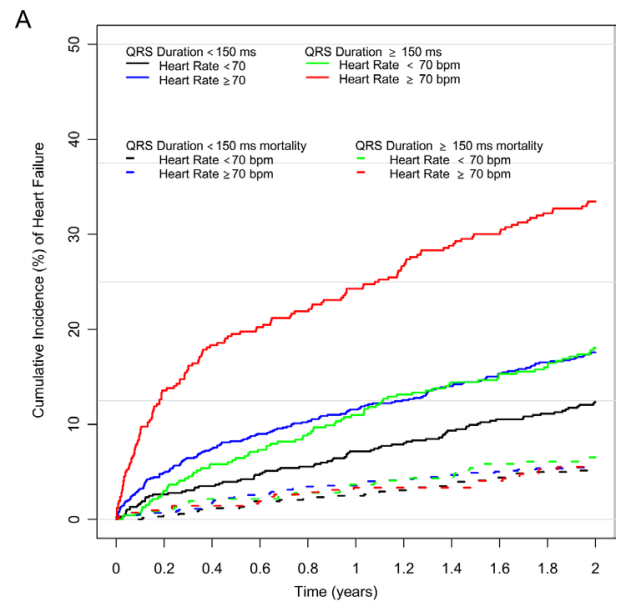

B

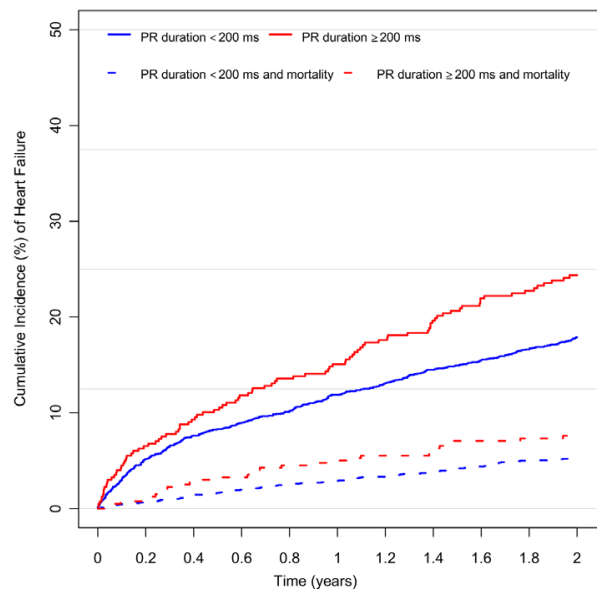

Figure 2 Cumulative incidence of heart failure by QRS duration and heart rate and by PR interval. Cumulative incidence of heart failure in patients with LBBB (A) with (1) QRS duration $<150 \mathrm{~ms}$ and heart rate $<70 \mathrm{bpm}$ (black line), (2) QRS duration $<150 \mathrm{~ms}$ and heart rate $\geq 70 \mathrm{bpm}$ (blue line), (3) QRS duration $\geq 150 \mathrm{~ms}$ and heart rate $<70 \mathrm{bpm}$ (green line), and (4) QRS duration $\geq 150$ ms and heart rate $\geq 70 \mathrm{bpm}$ (red line); and (B) patients with PR interval $<200$ ms (blue line) and with PR interval $\geq 200 \mathrm{~ms}$ (red line). Solid lines represent heart failure. Dotted lines represent death from other causes. bpm, beats per minute; LBBB, left bundle branch block.
Patients' baseline characteristics according to these subgroups are shown in table 1.

\section{Risk of HF based on QRS duration and heart rate}

Approximately $33 \%$ of patients with a QRS duration of $\geq 150 \mathrm{~ms}$ and a heart rate of $\geq 70 \mathrm{bpm}$ developed HF within 2 years compared with approximately $12 \%-18 \%$ in the other subgroups (figure 2A, table 1). When compared with the subgroup with a QRS duration $<150$ and heart rate $<70$, adjusted Cox regression analyses showed that all remaining subgroups had a significantly higher HR of $\mathrm{HF}$ (figure 3 ). The group with a QRS duration $\geq 150$ and a heart rate $\geq 70 \mathrm{bpm}$ had the highest HR 3.14 (95\% CI 2.38 to $4.13, \mathrm{p}<0.001)$.

Analyses of QRS duration as a continuous variable stratified by heart rate $(<70 \mathrm{bpm}$ and $\geq 70 \mathrm{bpm})$ using restricted cubic splines showed increasing hazard for HF along with increasing QRS duration, with a steeper incline among those with a heart rate $\geq 70 \mathrm{bpm}$ (figure $4 \mathrm{~A}, \mathrm{~B}$ ).

\section{Cumulative incidence and risk of HF by PR interval}

Patients' baseline characteristics according to PR subgroups are shown in online supplemental table 4 . Patients with a PR interval $\geq 200 \mathrm{~ms}$ were older $(76.0$ vs 71.3 ) and more likely to be men $(59.5 \%$ vs $34.9 \%)$, have hypertension $(47.5 \%$ vs $34.2 \%)$ and diabetes $(9.8 \%$ vs $7.0 \%)$. The incidence of HF was highest in the group with a PR interval $\geq 200 \mathrm{~ms}$ where approximately $24 \%$ of the patients with LBBB developed HF within 2 years compared with a PR interval $<200 \mathrm{~ms}$ where approximately $18 \%$ of the patients with LBBB developed HF within 2 years (figure 2B, online supplemental table 4).

A PR interval of $\geq 200 \mathrm{~ms}$ was associated with an HR of $1.40(95 \%$ CI 1.15 to $1.80, \mathrm{p}<0.01)$ compared with a reference PR interval of $<200 \mathrm{~ms}$ in unadjusted Cox analysis (figure 5). After adjustment, this association was no longer significant (HR of 1.16 (95\% CI 0.92 to 1.46), $\mathrm{p}=0.225$ ) (figure 5).

Modelling the PR interval as a continuous variable using restricted cubic splines showed no significant

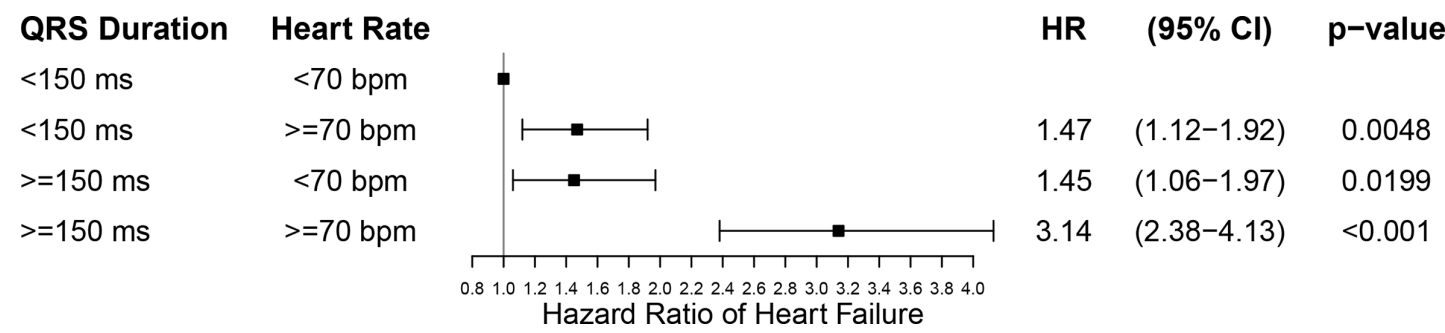

Figure 3 Risk of heart failure by QRS duration and heart rate. Multivariable Cox regressions showing $\mathrm{HRs}$ with $95 \% \mathrm{Cl}$ of associations between heart failure in patients with LBBB within four subgroups: (1) QRS duration $<150$ ms and heart rate $<70$ bpm, (2) QRS duration <150 ms and heart rate $\geq 70 \mathrm{bpm}$, (3) QRS duration $\geq 150 \mathrm{~ms}$ and heart rate $<70 \mathrm{bpm}$, and (4) QRS duration $\geq 150 \mathrm{~ms}$ and heart rate $\geq 70 \mathrm{bpm}$. Adjusted for age groups, sex, hypertension, diabetes, previous acute myocardial infarction, chronic ischaemic heart disease, renal disease, beta-blockers and PR groups. bpm, beats per minute; LBBB, left bundle branch block. 
A Heart Rate $<70$ bpm

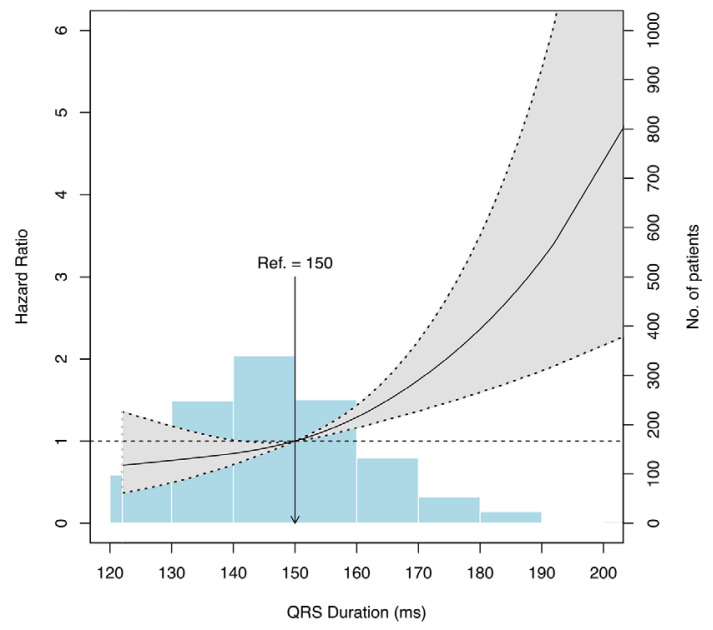

B

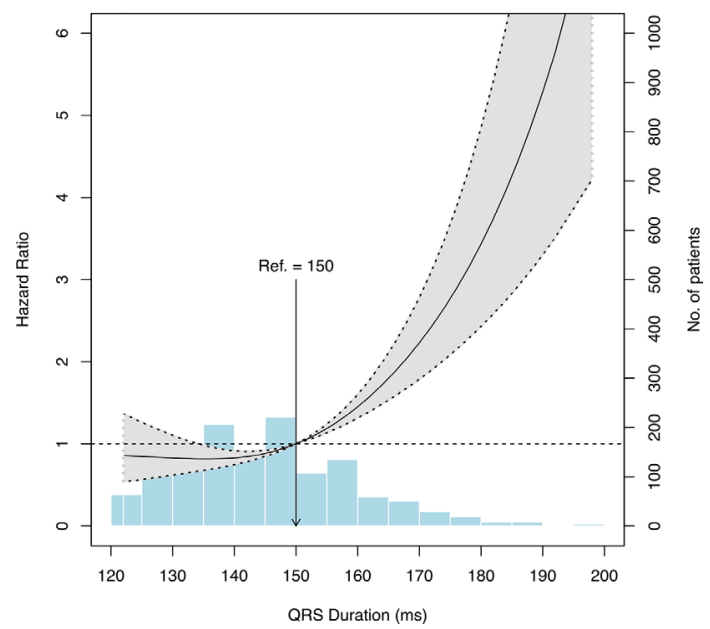

Figure 4 Association between QRS duration and the risk of heart failure by heart rate. Restricted cubic splines with three knots at 10th, 50th and 90th percentiles showing the association between QRS duration (continuous) and the risk of heart failure represented by $\mathrm{HR}$ and stratified by $(A)$ heart rate $<70 \mathrm{bpm}$ and $(\mathrm{B})$ heart rate $\geq 70 \mathrm{bpm}$. Grey area indicates $95 \% \mathrm{Cl}$. The reference values of $150 \mathrm{~ms}$ was used. The distribution of patients is represented by blue bars. Adjusted for age groups, sex, hypertension, diabetes, previous acute myocardial infarction, chronic ischaemic heart disease, renal disease, beta-blockers and PR groups. bpm, beats per minute. increase in HR for HF with increasing PR interval (online supplemental figure 1).

We observed no significant interactions between the PR groups and other covariates.

\section{Additional analyses}

Sensitivity analysis with $\mathrm{HF}$ and death as a combined endpoint revealed no notably changes of results in neither the QRS and heart rate subgroups nor the PR subgroups (online supplemental figures 2 and 3). Analyses with HF without the use of loop diuretics did not substantially alter results (online supplemental figures 4 and 5).

We stratified QRS and heart rate groups on the PR interval groups in multivariate analysis. These analyses revealed no statistically significant association with $\mathrm{HF}$ in any subgroup (data not shown).

Interaction analyses showed no significant interaction between any exposure and ischaemic heart disease, both with and without myocardial infarction.

\section{DISCUSSION}

Our study was based on a large number of patients with LBBB from the primary care sector. We found an interaction between QRS duration and heart rate on the risk of HF. The highest risk of HF was observed among subjects with a QRS duration $\geq 150 \mathrm{~ms}$ and a heart rate $\geq 70 \mathrm{bpm}$. No association between PR interval and the risk of HF was found. These findings from primary care setting ECGs hold potential for risk stratification and referral practice to secondary cardiology evaluation and care in patients with LBBB.

Many patients with LBBB are prone to dyssynchronous contraction due to prolonged activation time in presence of bundle block. A simultaneous increase in QRS duration will further accentuate this dyssynchronous, accelerating the impairment in LV systolic function and decline in LV ejection fraction, ${ }^{12}$ which in turn would cause the cardiovascular system to compensate. Compensatory mechanisms include a variety of factors, such as increased inotropic and chronotropic responses in order to increase the cardiac output. ${ }^{14}$ However, the persistent maintenance of the cardiac output creates a greater strain on the myocardium and promotes remodelling, which

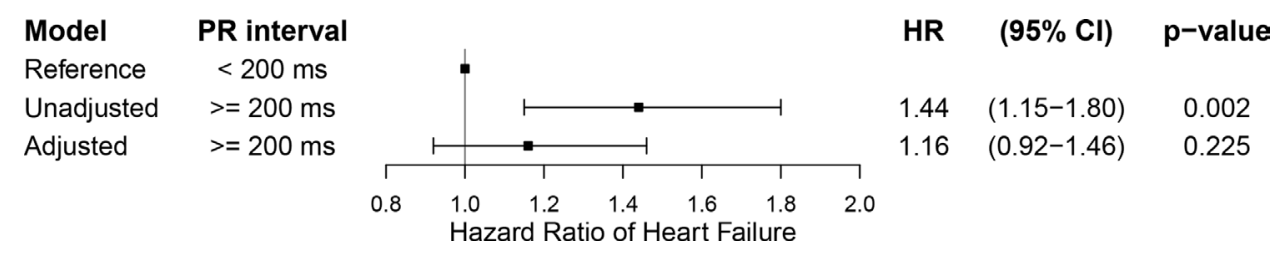

Figure 5 Risk of heart failure by PR interval. Multivariable Cox showing HRs with $95 \% \mathrm{Cl}$ of associations between heart failure in patients with LBBB with PR interval $<200 \mathrm{~ms}$ and $\geq 200 \mathrm{~ms}$, respectively. Adjusted for age groups, sex, beta-blockers, cardiac glycosides, calcium channel blockers and heart rate groups. LBBB, left bundle branch block. 
in turn deteriorates cardiac function. ${ }^{14}$ This duality of compensation and strain might explain the higher risk of $\mathrm{HF}$ in the groups with a heart rate $\geq 70 \mathrm{bpm}$. Our results are found in spite of the exclusion of patients with heart rate above $100 \mathrm{bpm}$, indicating that patients with LBBB with a higher heart rate, yet still in the normal range, should receive clinical attention.

We found no association between longer PR interval and HF in our study, implying that the PR interval holds no additional value in terms of HF risk in patients with LBBB. Similar results have been found by Aro $e t a l,{ }^{25}$ who found no increased risk of mortality or hospitalisation due to coronary artery disease, HF, atrial fibrillation or stroke with prolonged PR interval, and by Schneider $e t a l,{ }^{13}$ who in the Framingham Study, found that the PR interval in LBBB did not correlate with cardiovascular abnormalities. Similarly, Crisel $e t a l^{26}$ in the Heart and Soul Study found that first degree atrioventricular block was associated with increased risk of HF and mortality in patients with stable coronary artery disease. We hypothesised that since increased QRS duration may result in abnormal filling time of the ventricle, patients with prolonged QRS could be even more dependent on normal conduction from the sinus node to the ventricles. However, we did not observe any increased risk of $\mathrm{HF}$ with increased PR interval in patients with LBBB.

Finally, we found increased risk of HF with longer QRS duration, supporting previous findings. Dhingra $e t$ al found that increase in QRS duration was associated with increased risk of $\mathrm{HF}$ in patients with bundle branch block. ${ }^{8}$ Similarly, Zhang et al, in the Women's Health Initiative Study, found increased risk of HF with QRS duration 120-139 ms and even higher risk with QRS duration $\geq 140 \mathrm{~ms}$ in patients with LBBB. ${ }^{6}$ Likewise, Zhang et al found increased risk of $\mathrm{HF}$ in patients with ventricular conduction defects and QRS duration 120-139ms and even higher risk with QRS duration $\geq 140 \mathrm{~ms}$, when compared with no ventricular conduction defects (QRS $<100 \mathrm{~ms})^{11}$

Our results are similar to previous findings, showing that increasing QRS duration increases risk of HF. Our results also indicate that there are differences in the risk of HF within the group with LBBB according to QRS duration. Thus, our results support earlier studies regarding the importance of QRS duration in LBBB, but in addition, we present a new important parameter: heart rate. From our results, it is seen that prolonged QRS duration affects the myocardium differently in patients with LBBB . Hence, heart rate might be seen as a marker for the degree to which the myocardium has been affected by the prolonged QRS duration. QRS duration and heart rate, which are readily available from an ECG in the primary care setting where echocardiography and measurements of ejection fraction are not, are proposed as parameters for risk stratification of patients with LBBB.

Future studies should try to assess and confirm this interaction in regard to treatment, especially cardiac resynchronisation therapy in this group of patients.

\section{Limitations}

Even though our study is observational, the findings throughout were quite consistent. Despite our access to a large amount of data through Danish registries, some data are not recorded in these registries and were unavailable for use in this study. These include symptoms (New York Heart Association classification) and general practitioners' diagnoses, as well as clinical data such as echocardiographic data including LV ejection fraction, to confirm systolic functional impairment before, after and at the time of HF diagnosis, which are all important prognostic factors. Furthermore, we did not have information on indications for the ECG recordings. ECG screening of patients with no symptoms is not recommended. ${ }^{27} 28$ However, ECG recordings are widely used in the primary care sector as part of many routine examinations and one could argue that the population of this study probably is representative of patients with LBBB found by chance on primary care ECG recordings.

Newer, stricter criteria for LBBB proposed by Strauss $e t$ $a t^{29}$ were not available for analyses.

Our results are focused on the length of the QRS complex in patients with LBBB only, whereas other studies also include other ventricular conduction defects.

\section{CONCLUSION}

In patients with LBBB seen in the primary care sector, a longer QRS duration combined with a higher heart rate is associated with increased incidence and risk of $\mathrm{HF}$ compared with patients with less prolonged QRS duration. The length of the PR interval was not associated with increased risk of HF in patients with LBBB.

\section{Author affiliations \\ ${ }^{1}$ Department of Clinical Epidemiology, Aalborg University Hospital, Aalborg, Denmark \\ ${ }^{2}$ Department of Health, Science and Technology, Aalborg University Faculty of Health Sciences, Aalborg, Denmark \\ ${ }^{3}$ Department of Cardiology, Nephrology and Endocrinology, Nordsjaellands Hospital, Hillerod, Denmark \\ ${ }^{4}$ University of Copenhagen, Danish National Research Foundation Centre for Cardiac Arrhythmia (DARC), Copenhagen, Denmark \\ ${ }^{5}$ Department of Cardiology, Laboratory of Molecular Cardiology, The Heart Centre, Copenhagen University Hospital, Rigshospitalet, Copenhagen, Denmark \\ ${ }^{6}$ Department of Cardiology, Hospital Vendsyssel in Hjørring, Hjorring, Denmark ${ }^{7}$ Department of Clinical Epidemiology, Aalborg University Hospital, Aalborg, North Denmark Region, Denmark \\ ${ }^{8}$ Department of Cardiology, Nordsjællands Hospital, Copenhagen, Denmark ${ }^{9}$ Cardiology Clinic Heart-Lung, Aalborg University Hospital, Aalborg, Denmark \\ ${ }^{10}$ Department of Cardiology, Regional Hospital North Jutland, Hjorring, Denmark}

Contributors MMS - manuscript drafting, data analysis and interpretation, and critical revising. JR—manuscript drafting, data analysis and interpretation, and critical revising. KWB — manuscript drafting, data analysis and interpretation, and critical revising. $\mathrm{SMH}$-data analysis and interpretation, and critical revising. $\mathrm{JN}$ - manuscript drafting, data analysis and interpretation, and critical revising. CG - conception/design of the work, data analysis, data acquisition, data analysis and interpretation, and critical revising. AHP—conception/design of the work, data analysis and critical revising. JBN—conception/design of the work, data acquisition and critical revising. BT-conception/design of the work, data analysis and interpretation, and critical revising. CP-conception/design of the work and critical revising. CT-P—conception/design of the work, data acquisition, data analysis 
and interpretation, and critical revising. PS—conception/design of the work, data analysis and interpretation, and critical revising. KHK-conception/design of the work, data analysis and interpretation, and critical revising.

Funding This study was funded by Aalborg University Hospital.

Disclaimer No external organisations had influence on study design, data, the preparation of the manuscript or the decision to submit for publication.

Competing interests KHK reports to have received speaker's honoraria from Novartis and research grant from the Laerdal Foundation. SMH has received support from the Danish Heart Foundation, The Danish Foundation Trygfonden and AstraZeneca. PS reports having received research grants from GE Health Care and BIOTRONIK. CP reports receiving speaking fees from H Lundbeck.

Patient consent for publication Not required.

Ethics approval Registry-based studies do not require ethical approval in Denmark. The use of data from registries was approved by The Danish Data Protection Agency (approval number 2008-58-0028).

Provenance and peer review Not commissioned; externally peer reviewed.

Data availability statement Data may be obtained from a third party and are not publicly available. Data can be acquired through applications to the relevant authorities.

Open access This is an open access article distributed in accordance with the Creative Commons Attribution Non Commercial (CC BY-NC 4.0) license, which permits others to distribute, remix, adapt, build upon this work non-commercially, and license their derivative works on different terms, provided the original work is properly cited, appropriate credit is given, any changes made indicated, and the use is non-commercial. See: http://creativecommons.org/licenses/by-nc/4.0/.

ORCID iD

Marc Meller Søndergaard http://orcid.org/0000-0001-6059-4478

\section{REFERENCES}

1 Francia P, Balla C, Paneni F, et al. Left bundle-branch blockpathophysiology, prognosis, and clinical management. Clin Cardiol 2007;30:110-5.

2 Eriksson $\mathrm{P}$, Hansson $\mathrm{PO}$, Eriksson $\mathrm{H}$, et al. Bundle-branch block in a general male population: the study of men born 1913. Circulation 1998;98:2494-500.

3 Fahy GJ, Pinski SL, Miller DP, et al. Natural history of isolated bundle branch block. Am J Cardiol 1996;77:1185-90 http://www.ncbi.nlm. nih.gov/pubmed/8651093

4 Eriksson P, Wilhelmsen L, Rosengren A. Bundle-branch block in middle-aged men: risk of complications and death over 28 years. The primary prevention study in Göteborg, Sweden. Eur Heart $J$ 2005;26:2300-6.

5 Witt CM, Wu G, Yang D, et al. Outcomes With Left Bundle Branch Block and Mildly to Moderately Reduced Left Ventricular Function. JACC Heart Fail 2016;4:897-903.

6 Zhang Z-ming, Rautaharju PM, Soliman EZ, et al. Different patterns of bundle-branch blocks and the risk of incident heart failure in the women's health Initiative (WHI) study. Circ Heart Fail 2013;6:655-61.

7 Haataja P, Nikus K, Kähönen M, et al. Prevalence of ventricular conduction blocks in the resting electrocardiogram in a genera population: the health 2000 survey. Int J Cardiol 2013;167:1953-60.

8 Dhingra R, Pencina MJ, Wang TJ, et al. Electrocardiographic QRS duration and the risk of congestive heart failure: the Framingham heart study. Hypertension 2006;47:861-7.

9 Rasmussen PV, Skov MW, Ghouse J, et al. Clinical implications of electrocardiographic bundle branch block in primary care. Heart 2019;105:1160-7.
10 Zannad F, Huvelle E, Dickstein K, et al. Left bundle branch block as a risk factor for progression to heart failure. Eur $J$ Heart Fail 2007;9:7-14.

11 Zhang Z-M, Rautaharju PM, Prineas RJ, et al. Ventricular conduction defects and the risk of incident heart failure in the Atherosclerosis Risk in Communities (ARIC) study. J Card Fail 2015;21:307-12.

12 Dobrzynski H, Anderson RH, Atkinson A, et al. Structure, function and clinical relevance of the cardiac conduction system, including the atrioventricular ring and outflow tract tissues. Pharmacol Ther 2013;139:260-88.

13 Schneider JF, Thomas HE, McNamara PM, et al. Clinicalelectrocardiographic correlates of newly acquired left bundle branch block: the Framingham study. Am J Cardiol 1985;55:1332-8.

14 Mann DL, Bristow MR. Mechanisms and models in heart failure: the biomechanical model and beyond. Circulation 2005;111:2837-49.

15 GE Healthcare. MarquetteTM 12SLTM ECG Analysis Program. Physician's Guide.

16 GE Healthcare. MarquetteTM 12SLTM ECG analysis program. Statement of validation and accuracy.

17 Jensen JR, Kragholm K, Bødker KW, et al. Association between Twave discordance and the development of heart failure in left bundle branch block patients: results from the Copenhagen ECG study. J Electrocardiol 2019;52:39-45.

18 Shenkman HJ, Pampati V, Khandelwal AK, et al. Congestive heart failure and QRS duration. Chest 2002;122:528-34.

19 Kümler T, Gislason GH, Kirk V, et al. Accuracy of a heart failure diagnosis in administrative registers. Eur $J$ Heart Fail 2008;10:658-60.

20 Gjesing A, Gislason GH, Køber L, et al. Nationwide trends in development of heart failure and mortality after first-time myocardial infarction 1997-2010: a Danish cohort study. Eur J Intern Med 2014;25:731-8

21 Krogager ML, Torp-Pedersen C, Mortensen RN, et al. Shortterm mortality risk of serum potassium levels in hypertension: a retrospective analysis of nationwide registry data. Eur Heart $J$ 2016;65:ehw129.

22 Schmidt M, Schmidt SAJ, Sandegaard JL, et al. The Danish National Patient Registry: a review of content, data quality, and research potential. Clin Epidemiol 2015;7:449-90.

23 Olesen JB, Lip GYH, Hansen ML, et al. Validation of risk stratification schemes for predicting stroke and thromboembolism in patients with atrial fibrillation: nationwide cohort study. BMJ 2011;342:d124.

24 Remme WJ, Swedberg K, Task Force for the Diagnosis and Treatment of Chronic Heart Failure, European Society of Cardiology. Guidelines for the diagnosis and treatment of chronic heart failure. Eur Heart J 2001;22:1527-60.

25 Aro AL, Anttonen O, Kerola T, et al. Prognostic significance of prolonged PR interval in the general population. Eur Heart $J$ 2014;35:123-9.

26 Crisel RK, Farzaneh-Far R, Na B, et al. First-degree atrioventricular block is associated with heart failure and death in persons with stable coronary artery disease: data from the heart and soul study. Eur Heart J 2011;32:1875-80.

27 Priori SG, Blomström-Lundqvist C, Mazzanti A, et al. 2015 ESC guidelines for the management of patients with ventricular arrhythmias and the prevention of sudden cardiac death. Eur Heart $J$ 2015;36:2793-867.

28 , Curry SJ, Krist AH, et al, US Preventive Services Task Force. Screening for cardiovascular disease risk with electrocardiography: US preventive services Task force recommendation statement. JAMA 2018;319:2308-14.

29 Strauss DG, Selvester RH, Wagner GS. Defining left bundle branch block in the era of cardiac resynchronization therapy. Am J Cardiol 2011;107:927-34. 